\title{
PERFIL DE UMA UNIDADE INTENSIVA CORONARIANA QUE REALIZA MOBILIZAÇÃOPRECOCE FISIOTERÁPICA
}

Guilherme Henrique Dalaqua Grande, Débora Mayumi De Oliveira Kawakami, Ana Maria Carrilho, Gabriela Ribeiro Menosse, Susimaryaparecidatrevisan Padulla, Rafael Zambeli Pinto.

Universidade Estadual Paulista-UNESP, Faculdade de Ciências e Tecnologias, Departamento de Fisioterapia. Presidente Prudente, SP.

\section{RESUMO}

O objetivo do estudo foicoletar informações clínico-demográficas, dos prontuários de pacientes atendidos na unidade intensiva coronariana (UCO) de um hospital do município de Presidente Prudente - SP e descrever o trabalho de mobilização precoce realizado pela fisioterapia dentro dessa UCO. Trata-se de um estudo restrospectivo observacional descritivo, realizado com prontuários dos pacientes atendidos durante o período de setembro de 2013 à setembro de 2014. Foram extraídos os dados demográficos-clínicos, junto com o protocolo de mobilização precoce realizado na unidade composto por 5 fases. Neste período foram atendidos 697 pacientes, sendo que $42,9 \%$ eram do sexo feminino e idade média $67,8( \pm 13,1)$. Cada paciente recebeu em média 8,2 atendimentos fisioterápicos, sendo 1,7 mobilizações na fase 4 e 1,7 na fase 5 . No total $65 \%$ dos pacientes foram mobilizados na fase 4 e $43 \%$ dos pacientes mobilizados na fase 5 . Houve diferença entre o grupo Insuficiência Cardíaca Congestiva (ICC) e "outros" $(P<0.001)$. Verificou-se que as principais causas de internação apontam para as coronariopatias.

Palavras-chave: Unidades de Terapia Intensiva, Mobilização Precoce, Fisioterapia, Perfil Epidemiológico.

\section{PROFILE OF A CORONARY INTENSIVE CARE UNIT THAT CARRY OUT EARLY MOBILIZATION BY PHYSIOTHERAPISTS}

\begin{abstract}
Objective of the studyto collect demographic-clinical information from medical records of patients seen in the coronary intensive care unit of a hospital in PresidentePrudente - SP and describe the early mobilization work carried out by physiotherapy within that UCO. This is a descriptive observational retrospective study with medical records of patients seen during the period September 2013 to September 2014. The demographic-clinical information with the early mobilization protocol performed in the unit that consists of 5 stages. In this period 697 patients were seen, and $42.9 \%$ were female, average age $67.8( \pm 13,1)$. Each patient received an average of 8.2 physical therapy visits, 1.7 were mobilizations in phase 4 and 1.7 in phase 5 . In total $65 \%$ were mobilized in phase 4 and $43 \%$ of mobilized patients in phase 5 . There was difference between the Congestive heart failure group (ICC) and "other" $(P<0.001)$. The main causes of hospitalization point to coronary artery disease.

Key-words:Intensive Care Units, Early Ambulation, Physiotherapy, Epidemiological Profile.
\end{abstract}




\section{INTRODUÇÃO}

A evolução das unidades de terapia intensiva (UTI), aliados aos da ventilação mecânica e dos cuidados intensivos, permitem que pacientes criticamente enfermos possam se recuperar, aumentando assim a sobrevida desses pacientes ${ }^{1,2}$. Porém, atualmente, sabe-se que a imobilidade pode influenciar negativamente na recuperação de doenças críticas, devido às alterações sistêmicas associadas a ela, como doença tromboembólica, atelectasias, pneumônicas, úlceras de pressão, contraturas, alteração das fibras musculares de contração lenta para contração rápida, desmineralização óssea, atrofia e fraqueza musculoesqueléticas, além de poder afetar os barorreceptores, que contribuem para a hipotensão postural e taquicardia ${ }^{3,4}$.

Esse declínio pode ser atenuado por um programa de reabilitação física na UTI, favorecendo a restauração precoce dos sistemas corporais. Quando se fala em "precoce", referese ao conceito de que as atividades de mobilização começam imediatamente após a estabilização das alterações fisiológicas, e que inclui atividades terapêuticas progressivas, tais como exercícios de mobilidade no leito, sentado na beira do leito, em ortostase, transferência para uma poltrona e deambulação ${ }^{5}$.

Diante de tais constatações, o presente estudo objetivou coletar informações clínicodemográficas, dos prontuários médicos de pacientes atendidos na unidade intensiva coronariana em um hospital de Presidente Prudente-SP. O objetivo secundário deste estudo foi descrever o trabalho de mobilização precoce realizado pela fisioterapia, com a variedade de pacientes cardíacos.

\section{MATERIAIS E MÉTODOS}

Trata-se de um estudo retrospectivo observacional descritivo realizado no período de setembro de 2013 a setembro de 2014, em um hospital de PresidentePrudente-SP. O estudo foi realizado com os dados obtidos de prontuários dos pacientes atendidos na Unidade Intensiva Coronariana.

Todos os prontuários de pacientes atendidos durante o período de 13 meses na UCO foram incluídos no estudo. Os pacientes foram incluídos no estudo independentemente da idade, sexo, tipo de plano de saúde e diagnóstico. Apenas os prontuários de pacientes incompletos foram excluídos do estudo.

Todos os procedimentos utilizados foram aprovados pelo Comitê de Ética do Hospital e pelo Comitê de Ética em Pesquisa da Faculdade de Ciências e Tecnologia - FCT/UNESP (Proc. no 02592612.8.0000.5402) obedecendo a Resolução 466/2012 do Conselho Nacional de Saúde de $12 / 12 / 2012$.

\section{PROCEDIMENTOS E COLETA DE DADOS}

Foram analisadas e tabuladas as fichas utilizadas pelos fisioterapeutas do setor, que contém dados demográficos e clínicos dos pacientes. Os dados demográficos coletados incluem: nome, sexo, idade, plano de saúde e região residente.

Os dados clínicos coletados foram: estado geral do paciente, diagnóstico, fatores de risco e doenças associadas, tempo de permanência na unidade intensiva coronariana, número de atendimentos fisioterapêuticos recebidos e fases do protocolo de mobilização. Tais dados são coletados na entrada do paciente na unidade, concomitantemente com outros membros da equipe e é evoluído a cada atendimento pelo fisioterapeuta responsável. Os atendimentos fisioterapêuticos são realizados três vezes ao dia nos turnos da manhã, tarde e noite.

Os pacientes foram agrupados quanto ao seu diagnóstico médico em quatro grupos. Grupo 1 de Arritmias, Grupo 2 de Insuficiência Cardíaca, Grupo 3 de Coronariopatias que inclui, angina instável, angioplastia, infarto agudo do miocárdio, revascularização do miocárdio, entre outros e Grupo 4 de Outros, inclui patologias cardíacas não de base. 
O tempo de permanência na unidade intensiva coronariana foi calculado utilizando as datas de internação e alta ou óbito. Já o número de atendimentos fisioterapêuticos, fases do protocolo de mobilização precoce e descrição do estado geral do paciente foram registrados pelos fisioterapeutas em uma ficha específica, a cada atendimento.

\section{PROTOCOLO DE MOBILIZAÇÃO}

Após várias buscas em artigos e discussões a equipe do setor, instalou na unidade um protocolo de mobilização precoce, baseado em Christiane Perme e Rohini Chandrashekar ${ }^{15}$, e adaptado para os pacientes da UCO, ${ }^{6,7,8,9}$, sendo realizado com todos os pacientes que dão entrada no setor - seguindo alguns critérios de inclusão no momento do atendimento. O mesmo é composto por 5 fases progressivas, que podem ser interrompidas ou retardadas de acordo com a evolução do paciente - Fase 1: no leito, exercícios passivos (paciente inconsciente), Fase 2: no leito, exercícios motores e respiratórios ativos, Fase 3: na beira do leito mais os exercícios, Fase 4: mobilizar para a poltrona mais os exercícios e Fase 5: deambulação mais os exercícios.

\section{ANÁLISE DOS DADOS}

As variáveis epidemiológicas e clínicas foram analisadas de forma descritiva. Dados dicotômicos e categóricos, dados com distribuição normal e dados com distribuição não normais foram reportados utilizando frequência (proporção), média (desvio padrão) e mediana (intervalo interquartílico), respectivamente.

O teste de Pearson chi-square foi usado para investigar a diferença entre as fases do protocolo. Esta análise foi ajustada para o número de altas ocorrido em cada grupo diagnóstico. Para as comparações consideradas estatisticamente significativas, odds ratio foi calculado. Foi utilizado o software estatístico IBM SPSS versão 20.0 (IBM corporation, Somers, NY, USA).

\section{RESULTADOS}

Entre o período de setembro de 2013 e setembro de 2014, 710 pacientes deram entrada na UCO. Desse total de pacientes, 13 pacientes foram excluídos da pesquisa por apresentar prontuários incompletos.

Dos 697 incluídos, $42,9 \%$ eram do sexo feminino, com idade média de $67,8( \pm 13,1)$ anos. Do total de internações $39,9 \%$ era pelo SUS e $60,1 \%$ pelo convênio; com $46,6 \%$ pacientes residentes em Presidente Prudente e 42,3\% na região do Oeste Paulista, como mostra a tabela 1.

Tabela 01. Características da Amostra

\begin{tabular}{|c|c|c|c|c|c|}
\hline & Todos & Grupo 1 & Grupo 2 & Grupo 3 & Grupo 4 \\
\hline & $(n=697)$ & $\begin{array}{c}\text { Arritmia } \\
(n=88)\end{array}$ & $\begin{array}{c}\text { ICC } \\
(n=108)\end{array}$ & $\begin{array}{c}\text { Coronariop } \\
\text { atias } \\
(n=369)\end{array}$ & $\begin{array}{c}\text { Outros } \\
(n=132)\end{array}$ \\
\hline Idade media (DP) & $\begin{array}{c}67,8 \\
(13,1)\end{array}$ & $\begin{array}{c}68,9 \\
(14,4)\end{array}$ & $70,9(14,1)$ & $65,9(12,3)$ & $\begin{array}{c}70,0 \\
(12,9)\end{array}$ \\
\hline \multicolumn{6}{|l|}{ Sexo n (\%) } \\
\hline Feminino & $\begin{array}{c}299 \\
(42,9 \%)\end{array}$ & $\begin{array}{c}47 \\
(53,4 \%)\end{array}$ & $54(50,0 \%)$ & $149(40,4 \%)$ & $\begin{array}{c}49 \\
(37,1 \%)\end{array}$ \\
\hline Masculino & $\begin{array}{c}398 \\
(57,1 \%)\end{array}$ & $\begin{array}{c}41 \\
(46,6 \%)\end{array}$ & $54(50,0 \%)$ & $220(59,6 \%)$ & $\begin{array}{c}83 \\
(62,9 \%)\end{array}$ \\
\hline \multicolumn{6}{|l|}{ Plano de saúde $n(\%)$} \\
\hline sus & $\begin{array}{c}278 \\
(39,9)\end{array}$ & - & - & - & - \\
\hline Particular & $\begin{array}{c}419 \\
(60,1 \%)\end{array}$ & - & - & - & - \\
\hline \multicolumn{6}{|l|}{ Localidade n (\%) } \\
\hline Presidente Prudente & $\begin{array}{c}325 \\
(46,6 \%)\end{array}$ & $\begin{array}{c}39 \\
(44,3 \%)\end{array}$ & $55(50,9 \%)$ & $177(48,0 \%)$ & $\begin{array}{c}54 \\
(40,9 \%)\end{array}$ \\
\hline Oeste Paulista & $\begin{array}{c}295 \\
(42,3 \%)\end{array}$ & $\begin{array}{c}38 \\
(43,2 \%)\end{array}$ & $41(38,0 \%)$ & $159(43,1 \%)$ & $\begin{array}{c}57 \\
(43,2 \%)\end{array}$ \\
\hline Outros & $41(5,9)$ & $\begin{array}{c}11 \\
(12,5 \%)\end{array}$ & $12(11.2 \%)$ & $33(8,9 \%)$ & $\begin{array}{c}77 \\
(11,1 \%)\end{array}$ \\
\hline
\end{tabular}

$\mathrm{n}$, tamanho da amostra; DP, desvio padrão 
Como diagnóstico teve a prevalência do grupo de coronariopatias, seguido de outros, insuficiência cardíaca e arritmias. Em relação aos fatores de risco 52,1\% apresentaram hipertensão arterial; 20,5\% diabetes mellitus; 14,2\% ex-tabagismo e 13,1\% dislipidemia, apontados na tabela 2.

Tabela 02. Prevalência dos fatores de risco e doenças associadas, estratificado por diagnóstico

\begin{tabular}{|c|c|c|c|c|c|}
\hline & Todos & Grupo 1 & Grupo 2 & Grupo 3 & Grupo 4 \\
\hline & $(n=697)$ & $\begin{array}{c}\text { Arritimia } \\
(n=88)\end{array}$ & $\begin{array}{c}\text { ICC } \\
(n=108)\end{array}$ & $\begin{array}{l}\text { Coronariopatias } \\
\qquad(n=369)\end{array}$ & $\begin{array}{l}\text { Outros } \\
(n=132)\end{array}$ \\
\hline \multicolumn{6}{|l|}{ Fatores de risco/doenças associadas } \\
\hline Hipertensão Arterial & $363(52,1 \%)$ & $43(48,9 \%)$ & $48(44,4 \%)$ & $199(53,9 \%)$ & $73(55,3 \%)$ \\
\hline Dislipidemia & $91(13,1 \%)$ & $8(9,1 \%)$ & $14(13.0 \%)$ & $57(15,4 \%)$ & $12(9,1 \%)$ \\
\hline Diabetes Mellitus & $143(20,5 \%)$ & $12(13,6 \%)$ & $24(22,2 \%)$ & $85(23,0 \%)$ & $22(16,7 \%)$ \\
\hline Insuficiência Renal Crônica & $35(5,0 \%)$ & $2(2,3 \%)$ & $14(13,0 \%)$ & $12(3,3 \%)$ & $7(5,3 \%)$ \\
\hline Acidente Vascular Encefálico & $18(2,6 \%)$ & $3(3,4 \%)$ & $6(5,6 \%)$ & $4(1,1 \%)$ & $5(3,8 \%)$ \\
\hline $\begin{array}{l}\text { Revascularização do } \\
\text { Miocárdio }\end{array}$ & $42(6,0 \%)$ & $7(8,0 \%)$ & $5(4,6 \%)$ & $24(6,5 \%)$ & $6(4,5 \%)$ \\
\hline Ex-tabagista & $99(14,2 \%)$ & $9(10,2 \%)$ & $10(9,3 \%)$ & $63(17,1 \%)$ & $17(12,9 \%)$ \\
\hline Tabagista & $53(7,6 \%)$ & $5(5,7 \%)$ & $2(1,9 \%)$ & $34(9,2 \%)$ & $12(9,1 \%)$ \\
\hline
\end{tabular}

Dados apresentados em frequência e porcentagem

Por fim cada paciente recebeu em média $8,2(16,0)$ atendimentos fisioterapêuticos, sendo $1,7(3,6)$ na fase 4 e $1,7(3,4)$ na fase 5 . O tempo de internação foi de $3,0(2,0) \| Q$, sendo $87,9 \%$ de altas; $11,2 \%$ óbitos e $0,7 \%$ transferências.

Tabela 03. Número de atendimentos, mobilizações, altas, óbitos, transferências e tempo de internação.

\begin{tabular}{|c|c|c|c|c|c|}
\hline & Todos & Grupo 1 & Grupo 2 & Grupo 3 & Grupo 4 \\
\hline & $(n=697)$ & $\begin{array}{c}\text { Arritimia } \\
(\mathrm{n}=88)\end{array}$ & $\begin{array}{c}\text { ICC } \\
(n=108)\end{array}$ & $\begin{array}{l}\text { Coronariopatias } \\
\qquad(n=369)\end{array}$ & $\begin{array}{l}\text { Outros } \\
(n=132)\end{array}$ \\
\hline $\mathbf{N}^{*}$ atendimentos & $8,2(16,0)$ & $6,9(12,0)$ & $13,5(20,7)$ & $6,8(15,2)$ & $8,8(15,1)$ \\
\hline $\mathbf{N}^{\bullet}$ atendimentos mediana (IIQ) & $4,0(6,0)$ & $4,0(7,0)$ & $7,5(10,5)$ & $4,0(4,0)$ & $4,0(7,0)$ \\
\hline Mob. Fase 4 & $1,7(3,6)$ & $1,2(1,7)$ & $3,3(5,8)$ & $1,3(2,9)$ & $1,6(3,6)$ \\
\hline Mob. Fase 4 mediana (IIQ) & $1,0(1,0)$ & $1,0(1,0)$ & $1,0(2,0)$ & $1,0(1,0)$ & $0,0(1,0)$ \\
\hline Mob. Fase 5 & $1,7(3,4)$ & $1,5(3,6)$ & $2,7(3,8)$ & $1,5(3,5)$ & $1,2(2,8)$ \\
\hline Mob. Fase 5 mediana (IIQ) & $0,0(2,0)$ & $0,0(1,0)$ & $1,0(4,0)$ & $0,0(2,0)$ & $0,0(1,0)$ \\
\hline Dias de Internação, mediana (IIQ) & $3,0(2,0)$ & $3,0(2,0)$ & $4,0(3,7)$ & $3,0(2,0)$ & $3,0(3,0)$ \\
\hline Número de Alta & $613(87,9 \%)$ & $80(90,9 \%)$ & $86(79,6 \%)$ & $345(93,5 \%)$ & $102(77,3 \%)$ \\
\hline Número de Obito & $78(11,2 \%)$ & $7(8,0 \%)$ & $20(18,5 \%)$ & $24(6,5 \%)$ & $27(20,5 \%)$ \\
\hline Transferências & $5(0,7 \%)$ & $0(0,0 \%)$ & $2(1,9 \%)$ & $0(0,0 \%)$ & $3(2,3 \%)$ \\
\hline
\end{tabular}

Dados são representados como média (desvio padrão), a não ser quando indicados. Dados categóricos ou dicotômicos utilizado frequência e porcentagem

Também foi visto que durante o período de 13 meses, 65\% dos pacientes da unidade de terapia intensiva foram mobilizados na fase 4 e $43 \%$ dos pacientes da unidade na fase 5 . Não houve diferença entre a proporção de pacientes mobilizados na fase 4 e 5 quando comparamos os grupos arritmia, ICC e coronariopatias. A única diferença encontrada foi para a comparação entre o grupo ICC e "outros" ( $\mathrm{P}<0.001)$. O grupo de ICC foi mobilizado mais vezes para fase $4(\mathrm{OR}=5.6$, $95 \% \mathrm{Cl}: 2.7$ to 11.5$)$ e fase $5(\mathrm{OR}=3.2,95 \% \mathrm{Cl}: 1.7$ to 5.7$)$ do que os pacientes do grupo "outros". 
Tabela 04. Número de mobilizações na fase 4 e 5, estratificado por grupos.

\begin{tabular}{lccccc}
\hline & Todos & Grupo 1 & Grupo 2 & Grupo 3 & Grupo 4 \\
\hline & $(\mathrm{n}=567)$ & $\begin{array}{c}\text { Arritimia } \\
(\mathbf{n}=88)\end{array}$ & $\begin{array}{c}\text { ICC } \\
(\mathbf{n}=108)\end{array}$ & $\begin{array}{c}\text { Coronariopatias } \\
(\mathbf{n}=369)\end{array}$ & $\begin{array}{c}\text { Outros } \\
(\mathbf{n}=132)\end{array}$ \\
Número de pacientes mobilizados fase 4 & 455 & $54(61.4 \%)$ & 82 & $260(70.7 \%)$ & 58 \\
& $(64.1 \%)$ & & $(75.9 \%)$ & & $(44.6 \%)$ \\
\hline Número de pacientes mobilizados fase 5 & 299 & $33(37.5 \%)$ & 60 & $172(46.7 \%)$ & 34 \\
& $(42.1 \%)$ & & $(55.6 \%)$ & & $(26.2 \%)$ \\
\hline
\end{tabular}

Dados apresentados em frequência e porcentagem.

\section{DISCUSSÃO}

A partir da análise deste estudo clínico-epidemiológico em uma UCO observou-se a prevalência do sexo masculino com idade média de $67,8(13,1)$ anos. O diagnóstico principal foi a coronariopatia acometendo metade dos pacientes admitidos e o principal fator de risco associado foi à hipertensão arterial. 0 tempo de internação foi de 3 dias (+/- 2) com média de atendimento fisioterapêutico e aplicação do programa de exercícios de 8,2 $(16,0)$ atendimentos. Os resultados também demonstraram ampla utilização e aderência aos programas de mobilização precoce em UTI's, visto que aproximadamente dois terços dos pacientes foram mobilizados na fase 4 e um pouco mais de dois quintos foram mobilizados na fase 5 do programa.

Os achados sócio-demográficos e clínicos deste estudo são semelhantes aos encontrados em outras pesquisas realizadas com população semelhantes ${ }^{10,11,12}$. Já em relação à utilização do programa de mobilização precoce, estudos recentes demonstraram que este programa, mesmo sendo em pacientes críticos, é uma prática segura ${ }^{13,14}$. Estudos recentes evidenciaram que o desenvolvimento de fraqueza generalizada relacionada ao paciente crítico é uma complicação importante e comum em muitos pacientes admitidos em uma unidade de terapia intensiva (UTI), tendo incidência entre $30 \%$ a $60 \%$ destes pacientes ${ }^{15,16}$.

Para reduzir as complicações da imobilidade nestas unidades, a mobilização precoce já é uma prática bastante difundida com alterações positivas significativas. O protocolo aplicado possui atividades terapêuticas progressivas, tais como exercícios de mobilidade no leito, sentado na beira do leito, em ortostase, transferência para uma poltrona e deambulação ${ }^{3,5,17,18}$.

As fases 4 e 5 do programa de exercícios, que incluem sedestação, ortostatismo e deambulação, apresentam os maiores impactos sobre os sistemas do individuo, sendo responsáveis por adaptações nos sistemas músculo-esqueléticos, cardiovasculares e respiratórios através do aumento da demanda e, consequentemente do trabalho destes sistemas.

Esta pesquisa evidencia o papel fundamental exercido pelo fisioterapeuta nas Unidades de Terapia Intensiva, demonstrando a necessidade de adequação de todas as instituições que ainda não possuem este profissional em seu quadro de funcionários. Uma metanálise de Cortes et al $(2009)^{22}$ demonstrou uma tendência para redução da mortalidade em pacientes submetidos a mobilização precoce após infarto agudo do miocárdio (IAM), confirmando a segurança do protocolo para os pacientes.

\section{CONCLUSÃO}

Verificou-se que as principais causas de internação apontam para as coronariopatias, com fatores de risco a hipertensão arterial e diabetes mellitus. No período de 13 meses, uma proporção considerável de pacientes são mobilizados dentro da unidade de terapia intensiva coronariana, sem complicações. Parece não haver diferença na frequência da mobilização entre os grupos de arritmia, ICC e coronariopatias.

Portanto, o presente estudo demonstra que a mobilização precoce em Unidades de Terapia Intensiva é uma realidade, está amplamente difundida e apresenta resultados 
convincentes de sua eficácia e benefícios a todos os pacientes, mesmo os mais críticos.Porém, outros estudos são necessários para avaliar a efetividade de cada técnica aplicada, assim como a elaboração de protocolos e diretrizes para cada situação específica.

\section{REFERÊNCIAS}

Chiang, LI; Wang, LY; Wu, CP; Wu, HD; Wu, YT. Effects of physical training on functional status in patients with prolonged mechanical ventilation. Phys Ther. 2006;86(9):1271-81. https://doi.org/10.2522/ptj.20050036.

De Jonghe, B;Bastuji-Garin, S; Durand, MC;Malissin, I; Rodrigues, P; Cerf, C;Outin, H;Sharshar, T.Groupe de Réflexionetd'Etude des NeuromyopathiesenRéanimation. Respiratory weakness is associated with limb weakness and delayed weaning in critical illness. Crit Care Med. 2007;35(9):2007-15. https://doi.org/10.1097/01.ccm.0000281450.01881.d8

Korupolu, R.; Gifford, JM; Needham, D. Early Mobilization of Critically III Patients: Reducing Neuromuscular Complications After Intensive Care. ContemporaryCritical Care. 2009;Baltimore, v. 6, n. 9.

Brower, RG. Consequences of bed rest. Critical Care Medicine. 2009; Baltimore, v. 37, n. 10, p. 422-428.

Needham, DM; Truong, AD; Fan, E. Technology to enhance physical rehabilitation of critically ill patients. Critical Care Medicine. 2009; Baltimore, v. 37, n. 10, p. 436-441.

Diretriz de Reabilitação Cardíaca. Arq. Bras. Cardiol. [online]. 2005; 84(5): 431-440. ISSN 0066$782 \mathrm{x}$.

Gil, CA; de Brito, FS; Castro, I; Ribeiro, JP; Mastrocola, LE; Ghorayeb, N; Yasbek, P; Vivacqua, R; Meneghello, R; Silveira, WI. Reabilitação Pós- Infarto Agudo do Miocárdio. ArqBrasCardiol. 1995; 64(03): 289-96.

Titoto L, Sansão MS, Marino LHC, Lamari NM. Reabilitação de pacientes submetidos à cirurgia de revascularização do miocárdio: atualização da literatura nacional. ArqCiênc Saúde. 2005;12(4): 216-19.

Sarmento, GJV. Fisioterapia respiratória no paciente crítico: rotinas clínicas. Barueri, SP: Manole, 2005; 582 p.

Fernandes, MVB; Aliti, G; Souza, EN. Perfil de pacientes submetidos à cirurgia de revascularização miocárdica: implicações para o cuidado de enfermagem. Rev. eletrônicaenferm; dez. 2009;11(4)

Freitas ERFS de. Profile and severity of the patients of intensive care units: prospective application of the. RevLatAm Enfermagem. May-June 2010;18(3): 317-323. https://doi.org/10.1590/S010411692010000300004.

Freitas, EOA;Pitthan, LO;Guido, LA;Linch, GFC;Umann, J. Fatores de risco cardiovascular emumaunidade de cardiologiaintensiva. Rev. enferm. 2010; UFPE on line; 4(1): 188-194. 
Silva, APP; Maynard, K; Cruz, MR. Efeitos da fisioterapia motora em pacientes críticos: revisão de literatura. RevBras Ter Intensiva. 2010; 22(1):85-91 https://doi.org/10.1590/S0103$\underline{507 \times 2010000100014}$

Mascarenhas, CM; Gonçalves, VS. A segurança da mobilização precoce em pacientes críticos: uma revisão de literatura. Interfaces Científicas -Saúde e Ambiente. Aracaju. out 2012;V.01;N.01;p. 8391.

Ali, NA; O’Brien, JM; Hoffmann, SP; Phillips, G; Garland, A; Finley, JC;Almoosa, K;Hejal, R. Acquired weakness, handgrip strength, and mortality in critically ill patients. Am J RespirCrit Care Med. 2008;178(3):261-8. https://doi.org/10.1164/rccm.200712-18290C

Maramattom, BV;Wijdicks, EF. Acute neuromuscular weakness in the intensive care unit. CritCare Med. 2006;34(11):2835-41. Review. https://doi.org/10.1097/01.CCM.0000239436.63452.81.

França, DC; Apolinário, AQ; Velloso, M; Parreira, VF. Reabilitação pulmonar na unidade de terapia intensiva: revisão de literatura. Fisioterapia e Pesquisa, São Paulo, jan/mar. 2010;v.17, n.1, p.81-7.

Dantas, CM; Silva,PFS; Siqueira, FHT; Pinto, RMF; Matias, S; Maciel, C; Oliveira, MC; Albuquerque, CG; Andrade, FMD; Ramos, FF; França, EET. . Influência da mobilização precoce na força muscular periférica respiratória RevBras Ter Intensiva. 2012; 24(2):173-178. https://doi.org/10.1590/S0103$\underline{507 \times 2012000200013}$

Cortes OL,Villar , JC; Devereaux, PJ; DiCenso, A. Early mobilisation for patients following acute myocardiac infarction: A systematic review and meta-analysis of experimental studies International Journal of Nursing Studies 46 (2009) 1496-1504. https://doi.org/10.1016/j.ijnurstu.2009.03.012. 